[DOI: 10.24214/jecet.A.9.3.46170.]

Jaurnal of Environmental Science, Computer Science and Engineering \& Technology

An International Peer Review E-3 Journal of Sciences and Technology

Available online at www.jecet.org

Section A: Environmental Science

Research Article

\title{
Extraction, isolation and characterization of cellulose nanocrystals obtained from Ayous sawdust
}

\author{
${ }^{1,2}$ Tchigo Alifa*, ${ }^{1}$ Joseph Marie Sieliechi, ${ }^{2}$ Jean-Bosco Tchatchueng \\ ${ }^{1,}$ Laboratory of Active Substances and Pollution (LSAP), Department of Applied \\ Chemistry, National Advanced School of Agro-Industrial Sciences (ENSAI), P.O. Box: \\ 455, University of Ngaoundere, Cameroon. \\ 2. Laboratory of Industrial Process and Chemical Engineering (LPIC), National Advanced \\ School of Agro-Industrial Sciences (ENSAI), P.O. Box: 455, University of Ngaoundere. \\ Cameroon.
}

Received: 08 June 2020; Revised: 19 June 2020; Accepted: 28 June 2020

\begin{abstract}
The present study aimed to extract and isolate cellulose nanocrystals from Ayous (Triplochiton scleroxylon) waste sawdust. Cellulose from Ayous sawdust was obtained by sequential multi-stage chemical extraction and bleaching. After the cellulose extraction, high quality of nanocrystals of cellulose were then extracted by using chemical acid hydrolysis. The chemical compositions, and morphology of nanocrystal of cellulose were analyzed by using standard methods, Fourier-transform infrared spectroscopy (FTIR), X-ray diffraction (XRD), Scanning electron microscopy (SEM) and crystallinity index was determined. A cellulose content of $46.6 \%$ was obtained after sequential extraction from the sawdust. SEM images showed the rodlike shape of NCC. According to XRD results, the crystallinity index increased upon chemical extraction. The crystalline index of Ayous wood fiber is $62 \%, 68 \%$ for mercerized cellulose and $86 \%$ for nanocrystalline cellulose.
\end{abstract}

Keywords: Ayous Sawdust, Extraction, Nanocrystalline Cellulose, Crystallinity Index 


\section{INTRODUCTION}

For many sectors of industrial applications, such as food processing, automobile and pharmaceutical industries, the properties of polymers are modified by using reinforcements to optimize their properties by synthetic fibres, such as the aramide or glass fibres ${ }^{[1-2]}$. However, the use of these reinforcements has a negative impact on the environment and on man, leading to respiratory disorders, skin allergies, cancers in the latter ${ }^{[3]}$.

In order to address environmental concerns, whose stakes are aimed at reducing the ecological impact of certain materials as well as dependence on petrochemicals, research has been pushed into the development of new materials using plant-based substances ${ }^{[4]}$. Thus, plant fibres have attracted special attention because of their low cost, low density, their bioavailability and biodegradability ${ }^{[5]}$.

Among the plant fibres used, the most widely exploited in composite materials are the jute, the flax and the hemp ${ }^{[6]}$. However, the use of agricultural waste and the waste from the industrial exploitation of wood, as reinforcement fibres begins to arouse interest, but their use in the materials remains secondary because of their low reinforcement properties ${ }^{[7]}$.

The waste from the wood transformation, is a major raw material pool that needs to be valorised. In Cameroon, wood logging is about $6 \%$ of gross domestic product (GDP). Wood is the second export after oil ${ }^{[8]}$. Ayous, of scientific name Triplochiton scleroxylon and represents $37.2 \%$ of the exploitation, is the essence of the most exploited wood. Its transformation, generates nearly 92 million $\mathrm{m}^{3}$ of residues ${ }^{[9]}$.Wood is a composite material of a very heterogeneous, porous and highly hygroscopic character, whose natural fibres consist mainly of cellulose, hemicelluloses and lignin ${ }^{[10-11]}$. However, even if these fibres have little interest in being used as reinforcements in composites, the elements of the components can still be exploited.

Cellulose is a semi-crystalline polymer present in the plant fibres in the form of a fibre bundles. Each bundle is made up of a cluster of cellulose microfibres composed of crystalline parts and amorphous areas. The crystalline parts called cellulose nanocrystals (CNC), are the smaller sizes structural elements in the plant fibres. Their extraction could thus be an effective solution for the valorization of plant waste ${ }^{[5-12]}$. This work will aim to extract microcrystalline cellulose from sawdust, isolate cellulose nanocrystals and carry out a characterization of the Ayous fiber and the different extracts.

\section{MATERIALS AND METHODS}

2.1. Materials: The Ayous sawdust, of scientific name Triplochiton scleroxylon belongs to the Sterculeiaceae family. The sample used in this study was taken from a sawmill located in the "Petit marché" market of the city of Ngaoundere, in the Vina division of the region of the Adamawa, Cameroon. The $99.9 \%$ sodium pellets and $80 \%$ sodium chlorite were obtained from MERCK, the $98 \%$ acid sulphuric $99.8 \%$ toluene, $96 \%$ ethanol were obtained from Sigma Aldrich. All the chemicals purchased were used as received without further purification.

\section{Experimental Methods}

\section{A. Characterisation of plant material:}

For the determination of dry matter, Dry matter is determined from a raw sample $(\mathrm{M})$, which is introduced into a previously dried ceramic crucible, then weighs $\left(\mathrm{M}_{0}\right)$ and placed in an oven at $105^{\circ} \mathrm{C}$ for three days, until a constant weight is obtained. After cooling in a dryer, the crucible containing the 
dry matter is weighed $\left(\mathrm{M}_{1}\right)$. Finally, the dry mass $(\mathrm{Ms})$ of the cold sample is weighed. The percentage of dry matter (MS) is given by the formula:

$$
M S=\frac{M_{S}}{M} \times 100
$$

The humidity rate $(\mu \%)$, is then obtained from the equation below:

$$
\mu \%=\left[\left(M_{0}-M_{1}\right) / M_{0}\right] \times 100
$$

Mineral matter is the part of the remaining plant matter, once all organic matter is extracted as a result of total combustion of the combustible in air. It is determined by calcination in a mitten oven at $550^{\circ} \mathrm{C}$ for 8 hours of mass (Ms) of previously dried plant matter at $105{ }^{\circ} \mathrm{C}$ for three days. After cooling in a dryer, the mass of mineral matter $(\mathrm{Mm})$ is weighed. The percentage of mineral matter $(\mathrm{MM})$ is given by the formula:

$$
M M=\frac{M_{m}}{M_{S}} \times 100
$$

The organic matter content (MO), is given by the formula:

$$
M O=M S-M M
$$

\section{B. Cellulose extraction:}

The cellulose of Ayous sawdust was obtained by multi-stage sequential extraction in accordance with the work of Astruc et al. ${ }^{[12]}$ with some modifications.

- Extraction of fats and extractables from sawdust from ayous: The sawdust was previously washed with distilled water and dried at $105{ }^{\circ} \mathrm{C}$ in the oven for 24 hours. Waxes, resins, fats and tannins were removed by Soxhlet extraction. The extraction was carried out for six hours, by a mixture of ethanol/toluene solvent $1: 2(\mathrm{v} / \mathrm{v})$. The grease-free sample was washed with distilled water and ethanol and dried at $75^{\circ} \mathrm{C}$ in an oven.

- Alkaline extraction: The hemicelluloses of the degreased sample were eliminated by alkaline treatment with $20 \%$ (w/v) $\mathrm{NaOH}$ at room temperature under magnetic agitation for 24 hours. The sample is filtered and washed with distilled water to remove any trace of $\mathrm{NaOH}$ and dried in the oven at $60{ }^{\circ} \mathrm{C}$ for 24 hours.

- Cellulose delignification and bleaching: Lignin is eliminated by treatment with $10 \%(\mathrm{v} / \mathrm{v})$ sodium chlorite in reflux heating for 2 hours at $\mathrm{pH} 4$. Then the sample is treated with a mixture of $18 \% \mathrm{NaOH}$ solution $-\mathrm{H}_{2} \mathrm{O}_{2}, 2: 1(\mathrm{v} / \mathrm{v})$, at a temperature of $40{ }^{\circ} \mathrm{C}$ to remove the lignin residues still present in the cellulose. A colour change is observed to white, then the sample is filtered and rinsed with distilled water until a $\mathrm{pH}$ of 7 is obtained.

\section{Isolation of cellulose nanocrystals (CNC):}

The cellulose extracted from Ayous sawdust, was subjected to acid hydrolysis with sulfuric acid at a concentration of $62.5 \%(\mathrm{w} / \mathrm{w})$, with magnetic stirring at $70{ }^{\circ} \mathrm{C}$ for 50 minutes. After adding the acid, the mixture was stirred for 30 minutes and washed with distilled water several times, then centrifuged, 
in order to separate the crystalline part from the amorphous part dissolved in the water-acid mixture for 10 minutes at a speed of $12000 \mathrm{rpm}$. Washing was also repeated with ethanol.

After centrifugation, the resulting particles were washed for a third time by dialysis with polyethylene glycol (PEG), maintained at a constant $\mathrm{pH}$. The mixture was also placed under sonication at $30{ }^{\circ} \mathrm{C}$ for 20 minutes, in order to avoid overheating of particles that could cause desulfation of the sulfate groups present on the cellulose.

\section{Chemical characterization methods of extracts}

- Fourier transform infrared spectroscopy (FTIR): Fourier transform infrared spectroscopy (FTIR) is a characterization method which highlights the chemical functions present in the material. These chemical bonds are highlighted by their own vibrational absorption. The spectrometer used was the Thermo Scientific Nicolet 6700. The measurements were carried out in transmission between 400 and $4000 \mathrm{~cm}^{-1}$ on powders of nanocrystals of dried cellulose.

- X-ray diffraction: crystallinity index (C.I): The analysis of the isolated CNCs was carried out by X-ray diffraction (XRD) on a PANalytical X'pert MPD-PRO apparatus of BraggBrentano geometry $\theta-\theta$, equipped with a secondary monochromator, a changer for $3 \times 15$ samples and an $\mathrm{X}^{\prime}$ celerator detector with an angular range of $2 \theta=8-80^{\circ}$. The $\mathrm{Cu}-\mathrm{K} \alpha \mathrm{X}$-ray source is generated at $45 \mathrm{kV}$ and $40 \mathrm{~mA}(\lambda=0.15188 \mathrm{~nm})$. The crystallinity index is obtained by carrying out the ratio of the crystallized area to the total area:

$$
C . I=\frac{\text { Total area }- \text { Amorphous part area }}{\text { Total area }} \times 100
$$

- Morphological analysis by scanning electron microscopy (SEM): Image of the scanning electron microscopy (SEM), were recorded in reflection mode on a TESCAN Vega LMU device.

\section{RESULTS AND DISCUSSION}

(A). Chemical characteristics of sawdust from ayous: The results presented in table 1, show the variation in the performance of each of the main constituents of Ayous wood and represent the averages obtained after several (five repetitions) laboratory tests. In this section, humidity, $\mathrm{pH}$, ash content, extractables, hemicelluloses, lignin and cellulose.

With a humidity of $15.7 \%$, Triplochiton scleroxylon is a wood in the tropical forest area of Africa that is relatively light with an average density of $0.38 \mathrm{~kg} / \mathrm{m}^{3}$. It dries quickly when exposed to the open air ${ }^{[13]}$. Humidity rate roughly equal to the result observed by Saha Tchinda et al. ${ }^{[9]}$, on Ayous whose humidity was $12 \%$.

Similarly, for the extractible content the results observed are almost identical to Saha Tchinda's work, carried out in 2015 on Ayous wood whose extractible content was $0.7 \%$ obtained by soxhlet extraction with dichloromethane solvent and $0.8 \%$ with ethanol/toluene solvent mixture ${ }^{[9]}$. These results are consistent with the laboratory results for the Ayous sawdust sample to study in this work.

After extraction of the tannins, the degreased sample was subjected to a successive extraction of hemicelluloses, lignin and cellulose whose levels obtained are respectively $24.3 \%$ for hemicelluloses which represent all sugars such as: pentoses and hexoses found in wood; $28.3 \%$ for lignin which is the 
matrix or cement that binds the various constituent elements of wood and $46.6 \%$ for valorisable microcrystalline cellulose.

Table 1: Characterization of sawdust (Ayous)

\begin{tabular}{r|c}
\hline Characteristics & Values \\
Humidity & $(15.7 \pm 0.5) \%$ \\
Ash content & $(2.9 \pm 0.3) \%$ \\
Volatile material content & $(81.2 \pm 2) \%$ \\
$\mathbf{p H}$ & $5.7 \pm 0.1$ \\
Extractables content & $(0.8 \pm 0.2) \%$ \\
Cellulose content & $(46.6 \pm 0.4) \%$ \\
Lignin content & $(28.3 \pm 0.3) \%$ \\
Hemicellulose content & $(24.3 \pm 0.5) \%$ \\
\hline
\end{tabular}

With a total yield of $70.9 \%$ of holocelluloses, these results are in line with the work of Saha Tchinda who studied Ayous wood in $2015^{[9]}$. This similarity of results is also observed for the results of Mounguengui's work in 2016 on two tropical-type trees of the Cesalpine botanical family: with $46.4 \%$ in the sapwood and $46.9 \%$ in the heartwood ${ }^{[14]}$.

(B). Characterization of wood fibre, microcrystalline cellulose and nanocrystalline cellulose by Fourier-transform infrared spectroscopy (FTIR): Taking into account the main differences between the IR spectra of the material before and after extraction and isolation of cellulose nanocrystals.

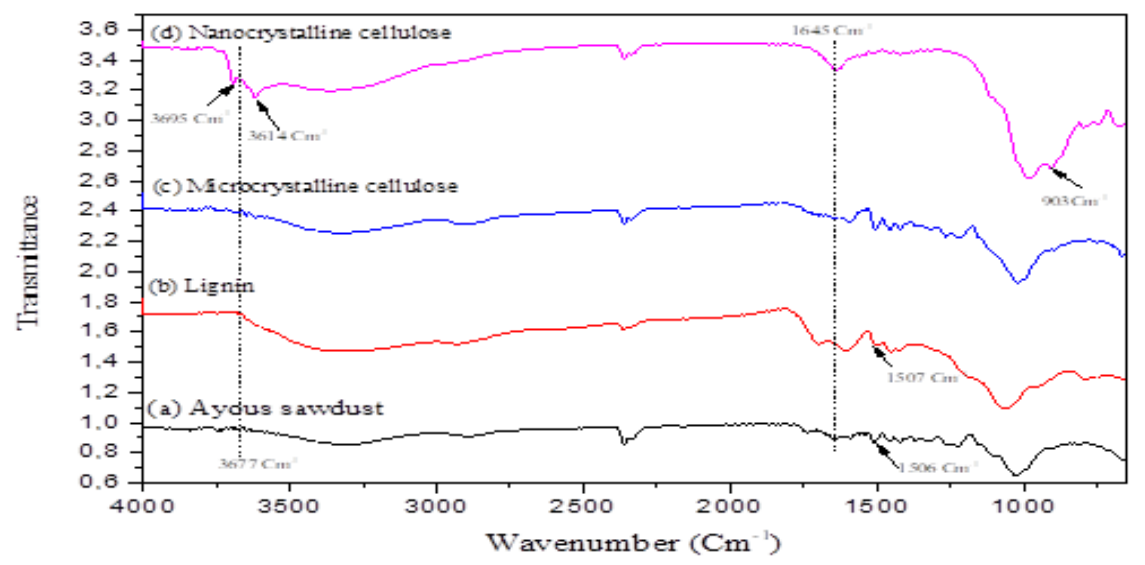

Figure 1: FTIR spectra of: (a) Ayous sawdust; (b) lignin; (c) microcrystalline cellulose; (d) nanocrystalline cellulose.

It is observed on Figure 1, that the FTIR spectra of sawdust, lignin and microcrystalline cellulose have the same form but with a decrease in absorption intensity, especially for mercerized microcrystalline cellulose obtained after multi-stage extraction on sawdust. However, a change in structure was observed in Figure 1 (d) for nanocrystalline cellulose with the appearance of new peaks. Three peaks were 
observed at $3695 \mathrm{~cm}^{-1}, 3614 \mathrm{~cm}^{-1}$ and $904 \mathrm{~cm}^{-1}$, this significant appearance of these peaks, was due to the elimination of the amorphous parts of the fiber, thus revealing the hidden functions, linked to the crystalline part of the cellulose.

The peaks of absorption in regions of 3600 to $3200 \mathrm{~cm}^{-1}$ and 1634 to $1591 \mathrm{~cm}^{-1}$ are attributed to the vibrations of stretching and bending, respectively, of the group's $\mathrm{H}_{2} \mathrm{O}$ and $\mathrm{OH}$. The peaks around $2900-2800 \mathrm{~cm}^{-1}$ correspond to a stretching $\mathrm{C}-\mathrm{H}^{[15-16]}$.

However, a decrease in the intensity of the absorption bands is observed, due to the elimination of extractives (especially waxes) with a characteristic absorption band at $2897 \mathrm{~cm}^{-1}$ associated with the $\mathrm{C}-\mathrm{H}$ stretching vibrations of the alkyl groups, hemicelluloses and lignin with a characteristic peak at $1507 \mathrm{~cm}^{-1}$, associated with C-C vibrations in aromatic rings and a $1224 \mathrm{~cm}^{-1}$ band associated with C-O bonds in carboxylic and ether groups.

This observation was also made by Abdul Rahman et al. ${ }^{[17]}$ on the extraction of cellulose from tea fibres with a characteristic spike of lignin observed for their work at $1508 \mathrm{~cm}^{-1}$. Similarly, in Figure 1, the peaks observed between 1430 and $1420 \mathrm{~cm}^{-1}$ and 1380 to $1330 \mathrm{~cm}^{-1}$ in all spectrums were attributed to the symmetrical flexion of $\mathrm{CH}_{2}$ and the bending vibrations of the $\mathrm{C}-\mathrm{H}$ of the aromatic rings in the polysaccharides, respectively ${ }^{[18]}$.

However, some peaks observed in the microcrystalline cellulose spectrum at 1339, 1318 and $893 \mathrm{~cm}^{-}$ ${ }^{1}$, compared to raw Ayous fibre and lignin indicate the presence of a crystalline cellulose structure of Type II and highlights the transformation of cellulose from type I to type II during the mercerisation procedure during which hemicelluloses and lignin were extracted from Ayous fibre ${ }^{[15-17]}$.

(C). Characterization of Ayous fibre, microcrystalline cellulose and nanocrystalline cellulose by X-ray Diffraction (XRD): On Figure 2, there is an evolution of the characteristic peaks of Ayous wood around $2 \theta=8^{\circ}, 16^{\circ}$ and $22.5^{\circ}$, significantly representing the characteristic peaks of native type-I cellulose. Generally, very few differences have been recorded between celluloses native to certain botanical sources such as: cotton, flax, hemp, rice husk or even wood fibre. The only difference observed is only significant peaks. For fibres with a high cellulose content, there are usually two peaks at $16^{\circ}$ and $22.5^{\circ}[17-19-20]$.

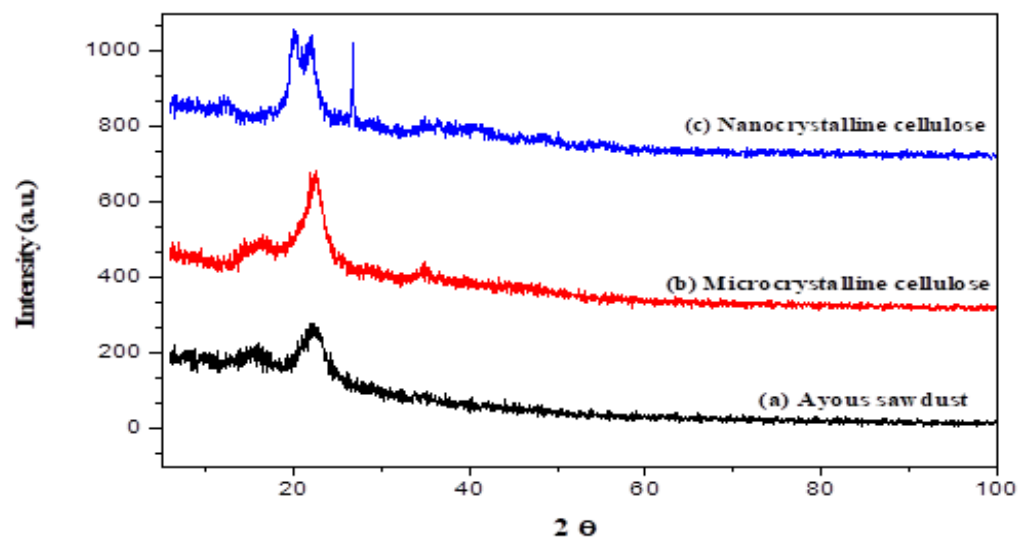

Figure 2: XRD Spectra of: (a) Ayous sawdust; (b) microcrystalline cellulose; (c) nanocrystalline cellulose

There was also an amplification of peaks around $2 \theta=14^{\circ}, 17^{\circ}, 22.5^{\circ}$ and $34^{\circ}$, for microcrystalline cellulose obtained, after extraction of hemicelluloses, lignin and bleaching. Ayous fibre is different from microcrystalline cellulose and nanocrystalline cellulose. The peak at $22.5^{\circ}$ is less pronounced for 
Ayous fibre, which possesses native mesh cellulose, monoclinic symmetry with crystallographic planes:

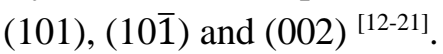

However, in microcrystalline cellulose, the peaks at $14^{\circ}$ and $17^{\circ}$ slightly shifted from the cellulose native to Ayous fibre, are difficult to dissociate because of the amorphous zones of microcrystalline cellulose. The latter is also related to crystallographic planes (101), (101) and (002). In addition, the peaks at $14^{\circ}$ and $17^{\circ}$ have completely shifted and merged to form a peak at two peaks characteristic of nanocrystalline cellulose. This crystalline form of cellulose is essentially synthesized by superior plants such as wood or cotton, which possess mostly native type-I $\beta$ cellulose ${ }^{[17-21]}$.

The crystalline index of Ayous wood fibre is 62\%, 68\% for mercerized cellulose and $86 \%$ for nanocrystalline cellulose. This variation reflects the amplification of the crystallinity of the cellulose obtained after progressive multi-stage elimination of the various constituents of the wood. The amorphous parts have been removed, thereby releasing the cellulose crystals. These results are consistent with observations made by Astruc et al. ${ }^{[12]}$ with a sharp increase in cellulose crystallinity due to sulphuric acid hydrolysis of the amorphous parts.

(D). Characterization of Ayous wood fibre, microcrystalline cellulose and nanocrystalline cellulose by scanning electron microscopy (SEM): Microscopic observation (Figure 3) of Ayous fibre, microcrystalline cellulose obtained by mercerization and nanocrystalline cellulose, showed that multi-stage extraction of Ayous wood fibre to alter the fibre structure of micro cellulose to cellulose nanocrystalline. In Figure 3 (A), Ayous fibre is organized into bundles of stratified structure with an association of several concentric layers called the wall. In general, the fibre consists of several bundles of cellulose microfibrils and they are covered with different amorphous layers (hemicelluloses and lignin) that act as cement and main matrix in the fibre ${ }^{[19]}$.
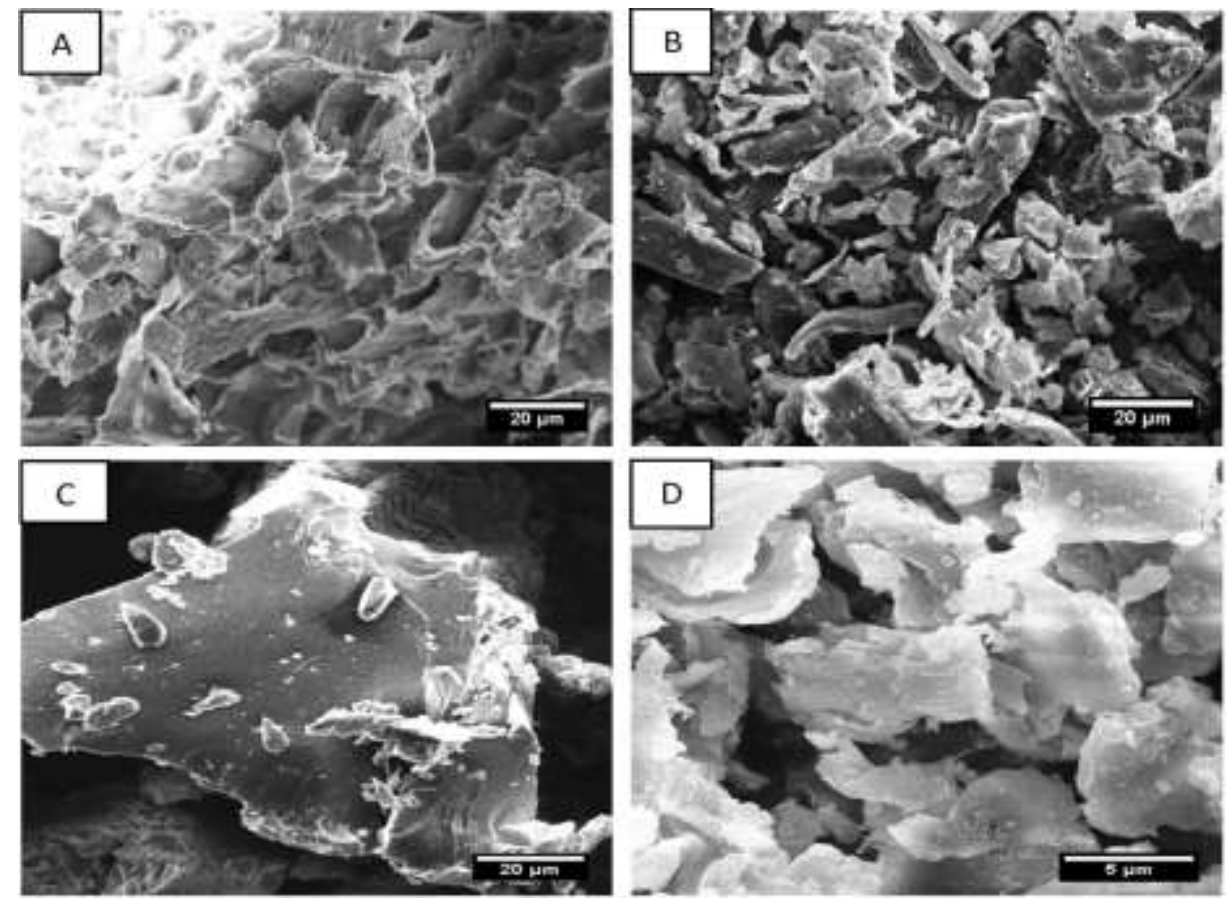

Figure 3: Microscopic structures of: Ayous wood fibre (A); Ayous microcrystalline cellulose(B); Ayous cellulose fibril (C); Ayous nanocrystalline cellulose (D) 
In Figure 3 (B), individual microcrystalline cellulose can be observed, due to the effective elimination of hemicelluloses and lignin because cellulose fibrils are incorporated into lignin and hemicelluloses. These allow the adhesion of microfibrils through non-covalent bonds ${ }^{[12-22]}$.

However, the surface of the microfibrils is still rough (Figure $3 \mathrm{C}$ ) due to the presence of the amorphous part in cellulose microcrystals. In figure 3 (D), microcrystalline cellulose has been subjected to acidic hydrolysis, clearly cellulose nanocrystal appears completely devoid of the amorphous part because sulphuric acid attacks the amorphous areas of cellulose by dissolution to release the crystalline part of type-II cellulose ${ }^{[19]}$.

\section{CONCLUSION}

During this work, the research focused first on extracting microcrystalline cellulose for the enhancement of waste from logging (Ayous fibre). 46.6\% cellulose yield, was obtained after elimination of tannins, hemicelluloses and lignin. Isolation of the cellulose nanocrystals by hydrolysis with sulfuric acid was carried out on the extracted microcrystalline cellulose, allowing the destruction of the amorphous zones by the attack of the protons released by the $\mathrm{H}_{2} \mathrm{SO}_{4}$. In addition, the results of the Infrared characterizations and X-ray diffraction, showed that the nanocrystals were effectively isolated after sequential extraction and chemical treatments. With $62 \%$ of crystallinity for Ayous sawdust, $68 \%$ for microcrystalline cellulose and $86 \%$ for nanocrystalline cellulose. The alkaline treatment during the extraction of hemicelluloses also allowed the formation of a type-II cellulose, thermodynamically more stable than the native type I cellulose from sawdust. The isolated nanocrystalline cellulose can be used for mechanical reinforcement and improve the hydrophilicity of organic membranes.

\section{REFERENCES}

1. D. Nabi Saheb, J.P. Jog, Natural Fiber Polymer Composites [J]. A Review Advances in Polymer Technology, 1999, 18 (4), 351-363.

2. J. Wu, D. Yu, C.-M. Chan, J. Kim, C.-M Mai, Effect of fiber pretreatment condition on the interfacial strength and mechanical properties of wood fiber/PP composites [J]. Journal of Applied Polymer Science, 2000, 76(7), 1000-1010.

3. L. Xue, L. G. Tabil, S. Panigrahi, Chemical Treatments of Natural Fiber for Use in Natural FiberReinforced Composites: A Review [J]. J. Polym Environ, 2007, 15, 25- 33.

4. Effect of acetylation on the properties of microfibrillated cellulose-LDPE composites Amaury Lepetit, Richard Drolet, Balázs Tolnai, Rachida Zerrouki, Daniel Montplaisir [J]. J. Appl. Polym. Sci., 2017, 134, 44933.

5. S. Thomas, S.A. Paul, L.A. Pothan, B. Deepa, Chapter 1: Natural Fibres: Structure, Properties and Applications: Cellulose Fibers: Bio- and Nano-Polymer Composites, Green Chemistry and Technology, Springer-Verlag, Berlin Heidelberg, 2011.

6. O. Faruk, A. K. Bledzki, H.-P. Fink, M. Sain, Biocomposites reinforced with natural fibers [J]. Prog. Polym. Sci.,2012, 37 (11), 1552-1596.

7. T. Väisänen, A. Haapala, R. Lappalainen, L. Tomppo, Utilization of agricultural and forest industry waste and residues in natural fiber-polymer composites: A review [J]. Waste Manag., 2016, 54, $62-73$. 
8. P.O. Cerutti, M. Mbongo, M. Vandenhaute, Report: State of the forest-wood sector in Cameroon, Food and Agriculture Organization of the United Nations and Center for International Forestry Research (CIFOR), Indonesia, 2016.

9. J.-B. Saha Tchinda, A. Pétrissans, S. Molina, M.K. Ndikontar, S. Mounguengui, S. Dumarçay, P. Gérardin, Study of the feasibility of a natural dye on cellulosic textile supports by red padouk (Pterocarpus soyauxii) and yellow movingui (Distemonanthus benthamianus) extracts [J]. Industrial Crops and Products, 2014, 60, 291-297.

10. A. Bledzki, J. Gassan, Composites reinforced with cellulose based fibres [J]. Progress in polymer science, 1999, 24, 221-274.

11. B. Agoudjil, A. Benchabane, A. Boudenne, L. Ibos, M. Fois, Renewable materials to reduce building heat loss: Characterization of date palm wood [J]. Energy and Buildings, 2010, 43, 491497.

12. J. Astruc, M. Nagalakshmaiah, G. Laroche, M. Grandbois, S. Elkoun, M. Robert, Isolation of cellulose-II nanospheres from flax stems and their physical and morphological properties [J]. Carbohydr. Polym., 2017, 178, 352-359.

13. Wageningen, Chapter 1 : Plantes médicinales 1, fondation Prota, ressources végétales de l'Afrique tropicale, Backhuys publishers/CTA, Pays-Bas, 2008, 276-281.

14. S. Mounguengui, J.-B. Saha Tchinda, M. K. Ndikontar, S. Dumarçay, C. Attéké, D. Perrin, P. Gérardin, Total phenolic and lignin contents, phytochemical screening, antioxidant and fungal inhibition properties of the heartwood extractives of ten Congo Basin tree species [J]. Annals of Forest Science, 2016, 73(2), 287-296.

15. H. Kargarzadeh, I. Ahmad, I. Abdullah, A. Dufresne, S.Y. Zainudin, R.M. Sheltami, Effects of hydrolysis conditions on the morphology, crystallinity, and thermal stability of cellulose nanocrystals extracted from kenaf bast fibers [J]. Cellulose, 2012, 19, 855-866.

16. D. Bagheriasl, P. J. Carreau, B. Riedl, C. Dubois, W. Y. Hamad, Shear rheology of polylactide (PLA)-cellulose nanocrystal (CNC) nanocomposites [J]. Cellulose, 2016, 23 (3), 1885-1897.

17. N.H. Abdul Rahman, B.W. Chieng, N. A. Ibrahim, N. Abdul Rahman, Extraction and Characterization of Cellulose Nanocrystals from Tea Leaf Waste Fibers [J]. Polymers, 2017, 9(11), 588.

18. M. Jonoobi, J. Harun, A.P. Mathew, M.Z.B. Hussein, K. Oksma, Chemical composition, crystallinity, and thermal degradation of bleached and unbleached kenaf bast (hibiscus cannabinus) pulp and nanofibers [J]. BioResources, 2009, 4(2), 626-639.

19. N.A. Rosli, I. Ahmad, I. Abdullah, Isolation and Characterization of Cellulose Nanocrystals from Agave angustifolia Fibre [J]. BioResources, 2013, 8, 1893-1908.

20. L. Segal, J.J. Creely, A.E. Jr. Martin, C.M. Conrad, An Empirical Method for Estimating the Degree of Crystallinity of Native Cellulose Using the X-ray Diffractometer [J]. Text. Res. J., 1959, 29, 786-794.

21. S. Kalia, B.S. Kaith, I. Kaur, Pretreatments of natural fibers and their application as reinforcing material in polymer composites: A review [J]. Polym. Eng. Sci., 2009, 49, 1253-1272. 
22. S. Beck-Candanedo, M. Roman, D.G. Gray, Effect of reaction conditions on the properties and behavior of wood cellulose nanocrystal suspension [J]. Journal of Biomacromolecules, 2005, 6, 1048-1054.

\section{* Corresponding Author: ${ }^{1,2}$ Tchigo Alifa*,}

1, Laboratory of Active Substances and Pollution (LSAP), Department of Applied Chemistry, National Advanced School of Agro-Industrial Sciences (ENSAI), P.O. Box: 455, University of Ngaoundere, Cameroun.

${ }^{2,}$ Laboratory of Industrial Process and Chemical Engineering (LPIC), National Advanced School of AgroIndustrial Sciences (ENSAI), P.O. Box: 455, University of Ngaoundere

Date of publication on line 28.06.2020 